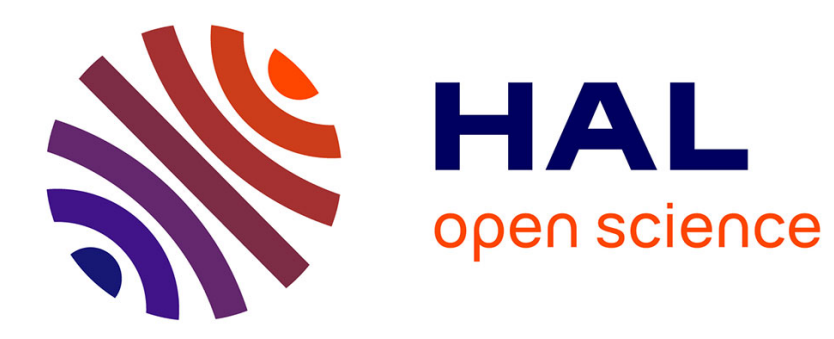

\title{
Introduction à la TMG et aux codes MMP
}

$\mathrm{S}$. Kiener

\section{- To cite this version:}

S. Kiener. Introduction à la TMG et aux codes MMP. Revue de Physique Appliquée, 1990, 25 (7), pp.737-749. 10.1051/rphysap:01990002507073700 . jpa-00246235

\section{HAL Id: jpa-00246235 https://hal.science/jpa-00246235}

Submitted on 1 Jan 1990

HAL is a multi-disciplinary open access archive for the deposit and dissemination of scientific research documents, whether they are published or not. The documents may come from teaching and research institutions in France or abroad, or from public or private research centers.
L'archive ouverte pluridisciplinaire HAL, est destinée au dépôt et à la diffusion de documents scientifiques de niveau recherche, publiés ou non, émanant des établissements d'enseignement et de recherche français ou étrangers, des laboratoires publics ou privés. 
Classification

Physics Abstracts

$41.10 \mathrm{H}-41.10-02.60-03.50$

\title{
Introduction à la TMG et aux codes MMP
}

\author{
S. Kiener \\ Institut für Feldtheorie, EPFZ, CH-8092 Zurich, Switzerland
}

(Reçu le 17 août 1989, révisé le 12 février 1990 et le 13 mars 1990, accepté le 23 mars 1990)

\begin{abstract}
Résumé. - L'ambition de ce papier est de présenter en langue française la TMG (Technique des Multipôles Généralisés, GMT en anglais) ainsi que les codes de calcul MMP (Multiple Multi-Pôles) d'une manière aussi complète que possible. Cette technique est basée sur les concepts suivants: (i) développement direct des champs électriques et magnétiques; (ii) choix de fonctions de base qui sont des solutions analytiques des équations de champs; (iii) application d'une technique de collocation généralisée, qui utilise un système surdéterminé d'équations résolu par la méthode des moindres carrés. Les avantages principaux des programmes sont: (i) pas d'équations intégrales à résoudre; (ii) discrétisation des seuls pourtours des domaines (même dans le cas des corps avec pertes) ; (iii) calcul précis des champs aussi bien dans les domaines ouverts qu'à la frontière des domaines fermés ; (iv) contrôle des résultats par une visualisation de l'erreur sur le pourtour du domaine ainsi que par la configuration des champs ; (v) efficacité des programmes existants notamment installés sur des PC et parallélisés afin d'utiliser des cartes avec plusieurs transputers (T800). Les principes analytiques et numériques de la méthode et des codes seront décrits d'une manière détaillée avec une courte comparaison avec d'autres méthodes. Les exemples qui seront finalement présentés dans le dernier paragraphe chercheront plus à montrer les possibilités des codes (vitesse et complexité) sans soucis particulier d'applications techniques précises.
\end{abstract}

Abstract. - The aim of this paper is to make a general presentation in French of the GMT (Generalized Multipole Technique) and of the computation codes MMP (Multiple MultiPoles). The basic concepts of this technique are : (i) direct expansion of the electric and magnetic field; (ii) a choice of basis functions which are analytical solutions of the field equations; (iii) application of a generalized point matching technique, which simply uses an overdetermined system of equations, which is solved in the least squares sense. The main advantages of the programs are : (i) no numerical integration; (ii) discretization of the boundaries only (even in the case of lossy materials) ; (iii) accurate computation of the fields in open domains and on boundaries of closed domains ; (iv) excellent control of the results with a plot of the errors on the boundary and a plot of the configuration of the fields ; (v) availability of efficient programs installed on PC's and parallelized for the use of boards of transputers (T800). Both analytical and numerical principles of the method and of the codes will be precisely described with a short comparison with other methods. In the last paragraph, the exemples have been chosen more to show the possibilities of the codes (speed and complexity) than in a concern of real technical applications.

\section{Introduction.}

Le calcul des champs électromagnétiques se fait maintenant essentiellement en utilisant des méthodes numériques, aussi nommées méthodes d'approximation. Pour l'utilisateur, il s'agit en priorité de choisir la «bonne " méthode, c'est-à-dire celle qui résout son problème, si possible dans un laps de temps raisonnable et de préférence pour un coût modéré. Cette évaluation, pourtant primordiale, n'est pas très aisée, car souvent l'information fournie par le titre de la méthode peut prêter à confusion. D’autre part, chaque méthode a ses avantages mis en avant par ses concepteurs, mais aussi ses inconvénients qui eux sont, en général, plus difficiles à découvrir. $\mathrm{Ce}$ fait, reconnu internationalement, conduit les producteurs de codes de calcul à définir des exemples canoniques ("Benchmarks") pour pouvoir ensuite faire une évaluation précise des différents codes. Les difficultés majeures rencontrées lors de l'élaboration des exemples montrent bien que personne n'est vraiment prêt à comparer « son » code avec celui des autres dans des conditions défavorables. 
Sous l'impulsion de A. Ludwig [14], différents groupes de par le monde (Confédération helvétique, USA, Japon, Israël) ont décidé de se réunir sous une bannière commune qui est le concept général TMG. Cette méthode, dont le développement dans notre groupe remonte à 1980 , a déjà été présentée à de nombreux congrès internationaux de langue anglaise ou allemande avec des résultats convaincants concernant des domaines aussi divers que les antennes, les guides d'ondes (lignes de transmissions, fibres optiques, etc.) ainsi que toute une série de problèmes variés en 2 et 3 dimensions. Les programmes ont un éventail très large et n'ont pas été développés pour un problème particulier. Ils se posent par leurs possibilités en concurrents de codes basés sur des méthodes comme EF (éléments finis) ou $\mathrm{MM}$ (méthode des moments). Ceci sera explicitement décrit dans un paragraphe séparé où une comparaison rapide avec différentes méthodes sera effectuée après la description complète des codes MMP.

Quant au but premier de cet article, il s'agit de montrer les principes théoriques et pratiques de notre méthode ainsi que ses performances à l'aide d'exemples choisis. Ceci pour favoriser l'utilisation des programmes déjà existants ou pour permettre la diffusion de codes basés sur cette méthode dans les régions francophones. Actuellement, la totalité de la littérature existante sur ce sujet est en allemand [2, 3,12 entre autres]. Les comptes rendus des conférences ainsi que les autres publications en anglais ou en français ne donnent que des éclaircissements sur des points particuliers et ne décrivent pas la méthode dans sa généralité $[4,8,11$ parmi de nombreuses autres].

La première partie sera dédiée aux principes théoriques aussi bien analytiques que numériques. Un court paragraphe sur une méthodologie permettant de comparer les différentes méthodes numériques conclura cette première partie.

La deuxième sera consacrée à la description de l'implantation des codes sur différentes machines ainsi qu'à son utilisation. Finalement, des applications seront présentées dans des domaines divers.

\section{Présentation des principes de la méthode}

Cette présentation se fera en deux parties, la première sera la description du chemin de résolution analytique et la seconde montrera le traitement numérique.

1. Partie analytique. - Soit les équations de Maxwell :

$$
\begin{aligned}
\operatorname{rot} \mathbf{H} & =\mathbf{j}_{0}+(\sigma-i \omega \varepsilon) \mathbf{E}=\mathbf{j}_{0}-i \omega \varepsilon^{\prime} \mathbf{E} \\
\operatorname{rot} \mathbf{E} & =i \omega \mu \mathbf{H} \\
\operatorname{div} \mathbf{H} & =0 \\
\operatorname{div} \mathbf{E} & =\rho / \varepsilon
\end{aligned}
$$

qui sont obtenues par l'utilisation de la loi d'Ohm $(\mathbf{j}=\sigma \mathbf{E})$, par les caractéristiques des matériaux $(D=\varepsilon E$ et $B=\mu H)$ et par la séparation de la fonction du temps $\left(f(t)=\mathrm{e}^{-i \omega t}\right.$, où $\omega$ peut être complexe). Après quelques manipulations mathématiques très classiques (et qui ne seront pas décrites ici), il est possible d'obtenir l'équation d'Helmholtz (aussi appelée, en Mathématiques, équation métaharmonique) pour des domaines linéaires, homogènes, isotropes et exempts de sources :

où

$$
\begin{gathered}
\left(\Delta+k^{2}\right)\left\{\begin{array}{l}
\mathbf{E} \\
\mathbf{H}
\end{array}\right\}=0 \\
k=\omega \sqrt{\varepsilon^{\prime} \mu} .
\end{gathered}
$$

Aux bornes des domaines, les conditions de continuité, dérivées des équations de Maxwell, doivent être respectées. Soit entre deux domaines $D_{i}$ et $D_{j}$ :

$$
\begin{aligned}
H_{\mathrm{t} i} & =H_{\mathrm{t} j} \\
E_{\mathrm{t} i} & =E_{\mathrm{t} j} \\
\mu_{i} H_{\mathrm{n} i} & =\mu_{j} H_{\mathrm{n} j} \\
\varepsilon_{i}^{\prime} E_{\mathrm{n} i} & =\varepsilon_{j}^{\prime} E_{\mathrm{n} j},
\end{aligned}
$$

où les lettres $\mathrm{t}$ et $\mathrm{n}$ désignent les composantes tangentielles ( 2 composantes) et normales à la frontière du domaine. Il faut remarquer que toutes ces conditions ne sont pas linéairement indépendantes les unes des autres, mais c'est un point qui sera développé dans la partie numérique.

A partir d'ici, la géométrie du problème à résoudre intervient. En effet, la signification de la géométrie et donc du système de coordonnées (ou de référence) joue un grand rôle. Les caractéristiques des opérateurs sont différentes dans chaque système de coordonnées (singularité pour l'opérateur de Laplace en coordonnées sphériques à l'origine $r=0$, etc.).

Les domaines d'application des codes MMP sont essentiellement des problèmes de guides d'ondes à symétrie cylindrique et d'antennes auxquelles un traitement en coordonnées sphériques est bien adapté. Ces deux systèmes de coordonnées correspondent à deux codes MMP, l'un en 2D (coordonnées $r, \phi, z$ ) et l'autre en $3 \mathrm{D}$ (coordonnées $r$, $\phi, \theta)$.

Pour le traitement en 2D, la séparation pour la composante de symétrie $z$ s'effectuera en prenant $f(z)=\mathrm{e}^{i \gamma z}$.

Il n'est évidemment pas souhaitable d'avoir à résoudre directement une équation vectorielle (Eq. (2.1)). On réduit donc l'équation vectorielle à une équation scalaire pour $E_{z}$ et $H_{z}$ pour ensuite déduire toutes les autres composantes à l'aide de ces deux seules composantes. C'est un calcul très classique qui découle directement des équations de Maxwell. Cette façon d'agir est d'autant plus intéressante que de nombreux cas pratiques se contentent d'un mode de propagation TE $\left(E_{z}=0\right)$ ou TM $\left(H_{z}=0\right)$. 
Il ne reste plus qu'à résoudre :

où

$$
\begin{gathered}
\left(\Delta+\kappa^{2}\right)\left\{\begin{array}{l}
E_{z} \\
H_{z}
\end{array}\right\}=0 \\
\kappa=\sqrt{k^{2}-\gamma^{2}}
\end{gathered}
$$

et $\gamma$ : constante de propagation, qui est une équation scalaire avec la valeur propre $\gamma$ à déterminer. Pour trouver une solution à ce problème, une méthode classique consiste à développer la fonction - dans chaque $D_{i}$ séparément - en une série de fonctions de base, par exemple, pour $E_{z}$ :

$$
E_{i z}=A_{i 0} f_{i 0}+\sum_{n=1}^{N} A_{i \mathrm{n}} f_{i \mathrm{n}}
$$

avec

$$
H_{i} f_{i \mathrm{n}}=0 \quad \text { et } \quad H_{i} f_{i 0}=g_{i}
$$

où $H_{i}$ est l'opérateur d'Helmholtz et $g_{i}$ la source (fonction d'inhomogénéité). Il est important de remarquer que c'est une expansion directe pour les champs électriques et magnétiques. $\mathrm{Ni}$ potentiel électrique, ni potentiel magnétique, ni courant ne sont nécessaires, ce qui évite de se poser le problème de la signification de la norme de Lorentz (Coulomb en statique). D'une manière générale, l'utilisation des potentiels $A$ (magnétique) ou $\phi$ (scalaire) n'apporte pas grand-chose dans le cas des champs dynamiques, car une équation d'Helmholtz doit toujours être résolue et de plus, ces grandeurs ne sont pas mesurables. Et surtout, il n'y a pas d'intégrales (type Coulomb) à calculer pour obtenir les champs électromagnétiques.

En trois dimensions, le calcul est tout à fait similaire. En partant de l'équation (2.1), on multiplie scalairement par $\mathbf{r}$ de deux côtés pour ensuite obtenir :

$$
\mathbf{r} \cdot\left(\Delta+k^{2}\right)\left\{\begin{array}{l}
\mathbf{E} \\
\mathbf{H}
\end{array}\right\}=\left(\Delta+k^{2}\right)\left(\mathbf{r} \cdot\left\{\begin{array}{l}
\mathbf{E} \\
\mathbf{H}
\end{array}\right\}\right)=0
$$

si, et seulement si $\operatorname{div} \mathbf{E}=\operatorname{div} \mathbf{H}=0$. Et, il ne reste plus qu'à résoudre, à nouveau, une équation scalaire d'Helmholtz pour $E_{r}$ et $H_{r}$, en séparant en modes TE

$$
r-r=\text {. }
$$

découlent des équations (1.1-4) écrites de la manière suivante :

$$
\begin{aligned}
\omega \varepsilon^{\prime} \mathbf{r} \cdot \mathbf{E} & =\frac{1}{i} \mathbf{r} \cdot(\boldsymbol{\nabla} \times \mathbf{H}) \\
& =\frac{1}{i}(\mathbf{r} \times \boldsymbol{\nabla}) \cdot \mathbf{H}=\mathbf{L} \cdot \mathbf{H} \\
\nabla \cdot \mathbf{H} & =0 \\
\omega \mu \mathbf{r} \cdot \mathbf{H} & =\frac{1}{i} \mathbf{r} \cdot(\boldsymbol{\nabla} \times \mathbf{E})=\mathbf{L} \cdot \mathbf{E} \\
\nabla \cdot \mathbf{E} & =0 .
\end{aligned}
$$

L'opérateur $\mathbf{L}$ est bien connu et ses composantes en coordonnées sphériques sont :

$$
\mathbf{L}=\left(\begin{array}{c}
0 \\
\frac{-1}{\sin \theta} \frac{\partial}{\partial \phi} \\
\frac{\partial}{\partial \theta}
\end{array}\right)
$$

avec

$\mathbf{L} \cdot \mathbf{L}=L^{2}$ : opérateur de Laplace angulaire .

Pour la résolution de l'équation (2.3), un développement similaire à celui en $2 \mathrm{D}$ sera effectué. Les fonctions de base sont choisies parmi les solutions analytiques des équations (2.1) ou (2.3). Ces fonctions sont appelées multipôles et sont continues, $n$ fois différentiables, linéairement indépendantes.

Un multipôle d'ordre $N$, en coordonnées sphériques, a la forme typique suivante :

$$
\begin{aligned}
& f_{i \mathrm{n}}(r, \theta, \phi)=\sum_{n=0}^{N} \sum_{m=0}^{M} \sqrt{\frac{\pi}{2 k_{i} r} H_{n+1 / 2}^{(1)}\left(k_{i} r\right) \times} \\
& \times P_{n}^{m}(\cos m \theta) \cos \phi
\end{aligned}
$$

où $H_{n+1 / 2}^{(1)}$ est une fonction de Hankel du premier ordre et $\boldsymbol{P}_{n}^{m}$ un polynôme de Legendre.

Le comportement de ces fonctions, qui sont d'un usage très souple et efficace, est essentiellement local (c'est-à-dire que leur valeur est grande dans le voisinage de leur origine et décroît exponentiellement avec l'éloignement). Ces fonctions sont calculées numériquement à l'aide de formules de récurrence, dont le temps de calcul - même s'il est plus important que pour des fonctions plus simples souvent utilisées par d'autres méthodes - reste raisonnablement réduit (pour des problèmes où le nombre d'inconnues est important, il devient même totalement négligeable).

Il se pose encore une question de pureté mathématique pour savoir si des solutions existent (mais la réalité physique nous donne la réponse). En revanche, la convergence des fonctions, quel que soit le pourtour étudié, est un problème difficile. I. N. Vekua [18] a traité le cas de l'équation métaharmonique d'une manière très complète. En utilisant les propriétés des fonctions holomorphes et en construisant les fonctions de Riemann correspondantes au problème, il résout (en deux dimensions) l'équation et prouve la convergence pour des domaines, dont le pourtour est lisse, même si c'est seulement au sens d'Hölder. Il existe donc une forte présomption de la justesse analytique de notre méthode.

En conclusion, la méthode est basée sur une résolution analytique des équations de Maxwell, en développant les fonctions de champs sur une base d'approximation. 
C'est maintenant la tâche du programme de déterminer les $A_{i}$ de l'équation (4).

2. PARTIE NUMÉriQue. - Ce paragraphe est luimême divisé en quatre sous-paragraphes dont le premier et le troisième sont essentiellement destinés aux utilisateurs. Le problème général sera de nouveau posé, d'une manière formelle, afin de permettre une classification des différentes méthodes numériques qui conclura cette première partie. Quant au deuxième, il montre un nouvel angle d'approche pour la TMG et donne le principe de fonctionnement des programmes ainsi qu'une description des contrôles internes des codes.

Chaque matériau physique (avec son $\varepsilon, \mu$ et $\sigma$ ) donnera naissance à un domaine. Il est parfois nécessaire de diviser un même matériau en plusieurs domaines pour faciliter le traitement de frontières aux formes très tarabiscotées. On parlera dans ce cas de (sous-)domaines fictifs, dont le traitement est exactement pareil aux autres.

Soit 2 domaines $D_{i}$ et $D_{j}$, le problème à résoudre se pose formellement, comme suit (problème de Dirichlet) :

$$
\begin{aligned}
H F_{i, j} & =G_{i, j} \\
\mathcal{H F}_{i, j} & =\mathcal{G}_{i, j} .
\end{aligned}
$$

L'équation (2i) représente les équations de champs d'Helmholtz homogènes $\left(G_{i, j}=0\right)$ ou pas. Nous avons vu dans la partie analytique que les fonctions de base sont des solutions des équations de champs. L'équation (3i) représente les conditions de continuité à la frontière des domaines. Ces conditions sont satisfaites numériquement dans un certain nombre de points du pourtour (matching points ou MP) en utilisant une généralisation de la technique collocation, qui grâce aux améliorations du père de la méthode, Ch. Hafner [2, 3], devient MMP. En revanche, dans d'autres groupes, l'évolution s'est plutôt faite au départ de la méthode des moments. A ce stade, il est important de préciser ce qui fait l'originalité des codes MMP. En effet, le traitement analytique n'apporte rien de franchement révolutionnaire, sauf si l'on considère que de nombreux codes calculent les différentes composantes des champs séparément. De même, le traitement numérique est connu (la méthode des moindres carrés remonte à Gauss) et le reste peut paraître de la cuisine de programmeur, si ce n'est qu'un certain nombre d'idées nouvelles ou plutôt la combinaison de toutes ces techniques permette ces performances des codes. Le fait d'utiliser des multipôles à la place de monopôles comme dans la méthode des charges [17] d'abord proposée par J. J. Thomson et dont les applications sont essentiellement en statique, d'utiliser des systèmes d'équations surdéterminés plutôt que des systèmes quadratiques comme dans la collocation classique (hypothèse de Rayleigh) et finalement d'autoriser plusieurs emplacements pour les multipôles permettant d'obtenir un meilleur traitement d'objets de forme variée. La méthode a pour point de départ les équations de Maxwell (et non les équations de Coulomb-Laplace comme dans la méthode des moments) et dérive d'une généralisation du principe de Huyghens où les sources du champ seraient placées à l'intérieur du corps.

Le système d'équations (2i) et (3i) permet une classification des différentes méthodes numériques:

- semi-analytique ((3i) satisfaite numériquement et (2i) analytiquement, par exemple TMG)

- semi-numérique ((2i) satisfaite numériquement et (3i) analytiquement)

- purement numérique (les équations sont approchées numériquement).

Les avantages et les inconvénients de chaque classe seront soulignés dans le dernier paragraphe, en rappelant que les méthodes purement analytiques n'ont plus qu'un champ d'application restreint.

2.1 Règles pour l'utilisateur. - Etudions maintenant, quelle est la marche à suivre pour résoudre un problème concret.

La première chose à faire est de simplifier la géométrie et d'engendrer les MP, sans oublier d'utiliser les symétries qui réduisent le coût du calcul. Le nombre total de MP est donné par la fréquence (un minimum de 5 et typiquement $10 \mathrm{MP}$ par longueur d'onde pour les surfaces courbes) et par le type de surface (avec ou sans discontinuité). Le choix de l'emplacement des MP est tout à fait libre (par exemple, équidistants les uns des autres), sauf cas très particuliers $[9,10]$. Comme les codes MMP sont basés sur l'utilisation de plusieurs multipôles par domaine, il faut déterminer l'origine de ces multipôles et bien choisir l'ordre maximum afin d'éviter des dépendances linéaires pour les fonctions dans un même domaine (et donc une matrice singulière). Ce processus a été complètement automatisé en 2D-statique [15] et en partie pour les codes dynamiques. Dans tous les cas de simples considérations géométriques sont suffisantes. Tout d'abord, comme l'équation (6) le montre, les multipôles ont une singularité à l'origine; il faut donc les placer à l'extérieur du domaine pour lequel ils fonctionnent ! Le concept des sphères tangentielles en $3 \mathrm{D}$ (cercles en $2 \mathrm{D}$ ) permet de couvrir le domaine à considérer.

La figure 1 montre comment le domaine extérieur à un cylindre métallique de longueur 5,5 unités et de diamètre 1 unité est approximé par trois sphères. La sphère supérieure est gardée ouverte afin de permettre une meilleure vue de l'angle de vision du MP. Les multipôles seront placés au centre des sphères. $\mathrm{Si}$ les sphères peuvent s'interconnecter, en aucun cas plusieurs multipôles ne peuvent être contenus dans une même sphère. Quant à l'ordre maximum, c'est aussi simple, puisqu'il s'agit de considérer l'angle de 


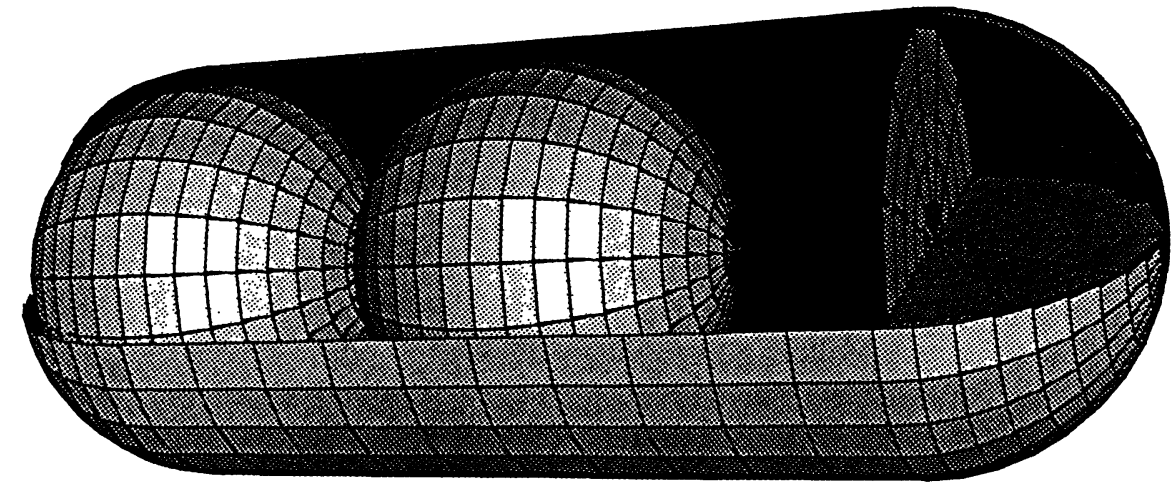

Fig. 1. - Cylindre métallique approximé par des sphères. [Metallic cylinder approximated by spheres.]

vision maximum (pour $\theta$ et $\phi$ ) du pôle et de deux MP voisins (dans la sphère d'influence du pôle, voir Fig. 1) :

$$
\begin{gathered}
n<\frac{\pi}{\alpha_{\theta_{\max }}} \\
l<\frac{\pi}{\alpha_{\phi_{\max }}} .
\end{gathered}
$$

Ceci permet d'une part d'obtenir un système qui soit suffisamment surdéterminé (4-10 fois plus d'équations que d'inconnues), le nombre d'équations étant donné par le nombre de MP (multiplié par le nombre de conditions de continuité, c'est-à-dire, en général, 3 pour les conducteurs parfaits ou 6 pour les autres cas). Le traitement des discontinuités (pointes, coins, etc.) demande un soin particulier dans le choix des MP et de l'ordre des multipôles $[9,10]$, dont l'emplacement est contrôlé d'une manière conventionnelle (ce sujet est vaste et ne sera pas traité dans le cadre de cette communication, il faut simplement remarquer qu'il est inutile d'implémenter des fonctions spéciales).

Pour des corps diélectriques avec ou sans pertes, le principe est le même, mais il est aussi possible d'utiliser comme fonctions de base ce que l'on appelle des "expansions normales » dont la forme est équivalente aux multipôles, si ce n'est que les fonctions de Hankel sont rem lacées ar des fonctions de Bessel. Ces fonctions ont une singularité à l'infini (si l'argument est complexe) et ne peuvent donc être utilisées que pour l'intérieur de corps. Les avantages et les limitations de cette technique seront décrits dans une publication ultérieure. Le cas général utilise donc des multipôles à l'extérieur du domaine diélectrique.

La figure 2 donne le comportement du champ électrique d'un dipôle.

Et ceci termine le travail de l'utilisateur ! S'il a respecté toutes ces règles, il n'y aura pas de problèmes de matrice mal conditionnée.

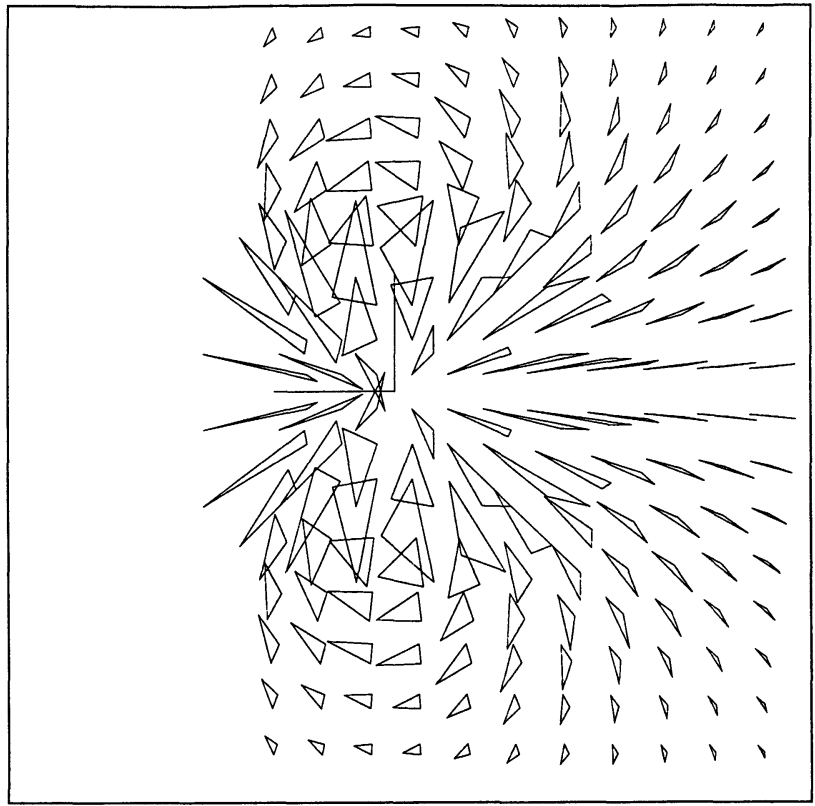

Fig. 2. - Champ électrique d'un multipôle sphérique d'ordre 0,1 .

[Electrical field for a multipole of order 0.1.]

2.2 Contrôles internes. - Etudions maintenant la méthode employée par le programme pour résoudre un problème. D'un côté la géométrie est définie et e autre un eve oppement en onc ion e ase es à disposition. Pour générer un système d'équations qui peut être résolu par un ordinateur, plusieurs possibilités s'offrent :

- Projection : un produit scalaire est défini, des fonctions tests sont choisies et on annule la projection de l'équation (de champ ou de continuité) sur les fonctions tests. Lorsque les fonctions tests sont égales aux fonctions de base, on parle de méthode de Galerkin.

- Collocation: pour chaque MP, on écrit les équations de continuité que l'on résout directement. 
Le grand désavantage de cette méthode est que, suivant les problèmes, l'emplacement des MP influence fortement la solution. D'autre part, si l'erreur est nulle à l'emplacement du MP, entre les MP c'est loin d'être le cas.

- Méthode de l'erreur (quadratique): il existe deux manières de présenter cette méthode qui se différencient essentiellement historiquement. Soit on imagine une généralisation de la collocation avec plus d'équations que d'inconnues ou l'erreur ne serait pas exactement nulle sur le MP, soit on définit une fonction erreur qui sera minimisée. Quoi qu'il en soit cette méthode est équivalente à la technique de projection avec un choix des fonctions tests de Galerkin en choisissant les "poids » correctement [3].

Considérons toujours la frontière $\partial G$ entre deux domaines $D_{i}$ et $D_{j}$ : soit $\eta^{2}$, la fonction d'erreur quadratique :

$$
\begin{gathered}
\eta^{2}=\eta_{G_{i}}^{2}+\eta_{G_{j}}^{2}+\eta_{\partial G}^{2}=\eta_{\partial G}^{2} \\
\eta_{\partial G}^{2}=\int_{\partial G}\left[\left|\mathbf{E}_{\mathrm{t} i}-\mathbf{E}_{\mathrm{t} j}\right|^{2}+\varepsilon_{i j}^{-2}\left|D_{\mathrm{n} i}-D_{\mathrm{n} j}\right|^{2}+\right. \\
\left.+Z_{i} Z_{j}\left(\left|\mathbf{H}_{\mathrm{t} i}-\mathbf{H}_{\mathrm{t} j}\right|^{2}+\mu_{i j}^{-2}\left|B_{\mathrm{n} i}-B_{\mathrm{n} j}\right|^{2}\right)\right] \mathrm{d} s
\end{gathered}
$$

où

$$
\begin{array}{r}
\varepsilon_{i j}=\sqrt{\varepsilon_{i} \varepsilon_{j}}, \quad \mu_{i j}=\sqrt{\mu_{i} \mu_{j}} \\
\text { et } Z_{i, j}=\sqrt{\mu_{i, j} / \varepsilon_{i, j}} .
\end{array}
$$

Une fois les composantes des champs écrites sous la forme d'une série où les $A_{i}$ sont inconnus, il suffit de différentier par rapport à ces coefficients et à annuler pour obtenir le système d'équations. Il est à remarquer que pour notre méthode «semi-analytique ", $\eta_{G_{i}}=\eta_{G_{j}}=0$ et seul le pourtour est à considérer. Cette fonction erreur a la dimension d'une énergie et un parallèle peut être tracé avec la méthode des EF. Dans sa classification des différentes méthodes numériques, Zhang $[19,20+$ livre à paraître] part du principe que toutes utilisent une fonctionnelle. Puis il distingue deux branches principales, l'une variationnelle et l'autre avec les résidus pondérés (Method of Weighted Residuals), puis il subdivise cette dernière entre méthode sur le pourtour et à l'intérieur du corps. Sur le pourtour existent essentiellement des méthodes intégrales (BIEM, BEM, etc.), mais aussi la TMG qui elle n'est pas une méthode intégrale. On peut aussi interpréter l'équation (8) comme le fait que les équations de continuité sont enregistrées, après avoir été dotées d'un poids approprié à l'emplacement de chaque MP. Cette nécessaire pondération des différentes équations s'explique par:

- Les dimensions de $E$ et $H$ sont différentes d'un facteur $10^{5}$, mais surtout $D$ et $E\left(\varepsilon_{0}=1 / 36 \pi \times\right.$ $\left.10^{-9}[\mathrm{MKSA}]\right)$ et $B$ et $H \quad\left(\mu_{0}=4 \pi \times 10^{-7}\right.$
[MKSA]) sont d'une dimension encore bien différente. Les équations de continuité devront être dotées d'un poids différent (voir Eq. (9)) pour éviter une prépondérance d'une des composantes du champ qui peut influencer la réalité physique du résultat.

- La distance au MP voisin définit aussi un poids pour chaque équation (ceci vient de l'équivalence avec la méthode de Galerkin).

- Et finalement, un poids supplémentaire peut être installé sur un MP particulier (pour que les conditions de continuité dans ce MP soient plus exactement remplies).

Tout ceci pour empêcher que des digits soient « effacés » lors du calcul.

Pour la mise en mémoire seul chaque rang est calculé, puis il est "update » et enregistré sous la forme d'une matrice triangulaire, avec un nombre de colonnes plus important que de lignes.

La résolution du système d'équations utilise la plus grande partie du temps de calcul. La recherche des valeurs propres (numériquement, à l'aide d'un algorithme d'approximation parabolique, par exemple), lorsque celles-ci sont complexes, peut s'avérer difficile, malgré le fait qu'une borne supérieure et inférieure soit donnée. Tout d'abord ce n'est pas un problème linéaire, ensuite il est difficile d'assurer l'unicité et la complétude des modes trouvés et finalement un minimum et non un zéro (dans le cas de MMP) est recherché. En passant, il faut remarquer que la valeur du déterminant n'est définie que pour une matrice quadratique. D'autre part, cette minimisation du "déterminant» (en fait, seul le dernier terme est considéré, car c'est la somme quadratique des erreurs) doit être effectuée soigneusement, sinon on obtient la solution triviale. Il faut donc normer la fonction erreur en la divisant par une amplitude : $\eta^{2} / A=$ minimum. Ceci permet aussi de privilégier un mode de propagation. Cette recherche peut s'effectuer interactivement en résolvant tout d'abord un problème équivalent, avec un $\gamma$ réel et en prenant cette valeur comme base de départ pour la seconde recherche (la plupart des applications pratiques utilisent des matériaux avec des pertes faibles).

Pour inverser une matrice non quadratique, la méthode la plus connue consiste à la multiplier par sa transposée et de l'inverser ensuite par une technique de Cholesky. C'est une méthode «bon marché » en temps de calcul, mais qui peut poser problème si la matrice n'est pas parfaitement conditionnée. Nous utilisons des routines LINPACK basées sur les plans de rotation de Givens (améliorées par une détection des zéros et une meilleure mise en mémoire) qui sont un peu moins rapides, mais plus fiables. Elles sont faciles à paralléliser, ce qui permet l'utilisation de cartes avec plusieurs «transputers » (T800) qui réduisent fortement (voir chapitre suivant) le temps de calcul. 
2.3 Dépendances linéaires, combinaisons et symétries. - Comment éviter les dépendances linéaires et les matrices mal conditionnées dans le cas d'un problème avec une géométrie complexe et qui demande de nombreux multipôles?

Ce n'est pas l'utilisation des 6 conditions de continuité qui pose problème car la dépendance linéaire analytique et numérique n'est pas équivalente. Bien au contraire, l'expérience a prouvé que ces conditions de continuité apportent un meilleur résultat [3]. Ceci est dû au fait que dans certaines configurations de champ, seule une des composantes est vraiment importante (par son amplitude). Si cette condition de continuité pour cette composante n'est pas explicitement remplie, alors la qualité du calcul n'est pas garantie. Quant aux différents ordres des multipôles, ils sont orthogonaux les uns aux autres et linéairement indépendants. En revanche, il y a des limitations à la taille des problèmes que l'on peut calculer. Tout simplement par la place mémoire disponible. Cela commence avec la limitation à $512 \mathrm{kB}$ des premières machines fonctionnant sur MS-DOS qui autorisait environ 120 inconnues, à des limites nettement plus élevées comme on le verra dans le chapitre suivant. Il existe pourtant plusieurs moyens pour réduire les dimensions de la matrice. L'un d'eux consiste d'abord à calculer un problème plus simple et ensuite d'utiliser ce résultat comme source du problème plus complexe. Un autre consiste à utiliser les symétries des corps et des champs. Le but avoué était aussi de gagner du temps de calcul, mais il s'est avéré que l'implantation de toutes sortes de symétries alourdissait le code et surtout s'est révélé d'un usage peu souple pour les utilisateurs. Actuellement, seules les symétries diadiques par rapport à chacun des plans $x y, x z$, $y z$ ont été conservées, même la symétrie rotationnelle en 3D n'est plus utilisée que pour des tests de vitesse.

Si l'on recherche la plus grande précision possible, il peut être utile de diviser certains matériaux en sous-domaines fictifs. D'autre part, il est facile d'implanter d'autres éléments: par exemple, on peut calculer le champ d'une antenne mince avec MM et ensuite étudier le champ de diffraction produit sur un autre objet avec MMP. D'autres applications utilisent les coordonnees cartesiennes ou encore des solutions analytiques (par exemple, les guides d'ondes rectangulaires, ou les modes TEM sur un câble coaxial) comme source de problèmes modifiés.

De plus, l'implantation des séries de Fourier permet le calcul de sources non sinusoïdales, notamment de trains d'impulsions. Les intégrales de Fourier (ou transformation de Laplace) posent le problème de la transformation inverse qui demande des temps de calcul importants.

D'autres exemples de combinaison avec d'autres méthodes sont en cours actuellement, un exemple est donné dans [11]. D'autres projets sont en cours afin de calculer des éléments non linéaires, etc.

2.4 Comparaison avec les méthodes les plus courantes. - Ce court paragraphe voudrait montrer les différences majeures qui existent entre les codes MMP et les autres codes basés sur d'autres méthodes. Nous nous limiterons à une comparaison avec les méthodes les plus usuelles comme la GTD (General Theory of Diffraction), MM (Méthode des Moments), EF (Eléments Finis) FD-TD (Finite Difference-Time Domain) et bien sûr la TMG. Il ne s'agit pas ici de décrire en détail chaque méthode, mais juste d'en indiquer les principes philosophiques principaux.

- GTD est basée sur la théorie des rayons lumineux qui remonte à Newton et à Fermat. Cette méthode utilise essentiellement des principes géométriques pour prévoir les réflections et son domaine d'application est en haute fréquence pour le calcul de grands objets.

- EF est apparenté à la physique quantique (Hamilton) par l'intégrale de variation qui est le cœur des codes. Bien adaptée aux domaines finis, non linéaires et de géométries complexes.

- FD-TD est basée sur les Mathématiques discrètes (discrétisation de l'opérateur) et le champ d'application est similaire à celui de la FEM.

- MM au sens de Harrington [6] utilise des principes que Coulomb et Ampère avaient développés, des équations intégrales et développe les courants et les charges. Il est particulièrement bien adapté aux objets fins et bons conducteurs.

- TMG résout l'équation d'Helmholtz et donc les équations de Maxwell. Il s'agit donc d'équations à dérivées partielles. Par rapport à GTD, les limites sont la taille du corps. Le problème pour toutes les méthodes basées sur la théorie ondulatoire de la lumière, ce sont les problèmes d'effacement de digits, car pour les hautes fréquences les ombres derrière le corps sont très franches et il est difficile pour un ordinateur d'obtenir un zéro absolu lorsque celui-ci est une superposition de plusieurs valeurs de fonctions.

avantage e et - sur a es que les non-linéarités peuvent être calculées. En revanche, les temps de dressage de la matrice sont importants. De plus les domaines ouverts demandent un traitement spécial. La situation actuelle est qu'il n'existe pas vraiment de codes EF capables de calculer les champs électrodynamiques non linéaires avec effet d'hystérésis.

MM et TMG ou plutôt NEC et MMP ont, en fait, de très grandes similitudes, mais un certain nombre de choix de base sont différents. Le tableau I donne plus de renseignements. 
Tableau I. - Sur ce sujet, il existe aussi un excellent article [16] qui compare les principes de plusieurs méthodes et en particulier discute de la validité de la MM.

\begin{tabular}{|c|c|c|}
\hline & MM-NEC & TMG-MMP \\
\hline $\begin{array}{l}\text { Equ. de champs } \\
\text { Equ. de continuité } \\
\text { Fonctions primaires } \\
\text { Fonctions de base } \\
\text { Discrétisation }\end{array}$ & $\begin{array}{l}\text { Coulomb, Ampère } \mathrm{E} \\
\text { et } \mathrm{H}, \text { eV d'autres } j \\
\text { ou } Q \\
\text { Fonctions « sim- } \\
\text { ples " suivant } \\
\text { matériau }\end{array}$ & $\begin{array}{l}\text { Helmholtz, Maxwell } \\
\mathrm{E} \text { et } \mathrm{H}, \mathrm{eV} \text { d'autres } \\
E \text { et } H \\
\text { Sol. anal. (multi- } \\
\text { pôles) pourtour seul }\end{array}$ \\
\hline
\end{tabular}

\section{Implémentation des programmes et exemples}

Un premier paragraphe montrera l'éventail actuel des programmes existants en décrivant le matériel utilisé. Finalement une série de trois exemples sera présentée.

1. LES PROGRAMMES MMP. - Les programmes MMP sont actuellement installés sur des ordinateurs personnels compatibles IBM-PC et sur des stations de travail SUN 3 et 4 . Les programmes sont écrits en Fortran. Pour des raisons de compilateurs, tous les programmes sont écrits en réels pour profiter de la double précision et bénéficier de la souplesse nécessaire d'implémentation (par exemple, le compilateur Fortran-3L ne soutient pas les complexes double précision). Nous nous sommes rendus compte que cela faisait dans tous les cas gagner (sic !) du temps de calcul, quelquefois dans des proportions vraiment intéressantes (sur SUN, le rapport de vitesse entre le programme utilisant les complexes double précision et la version en réels, est de 2 à 3 ).

L'installation sur de plus gros ordinateurs ne pose donc pas de problèmes, mais c'est uniquement une question de puissance de calcul qui peut justifier ce choix. Depuis que nous possédons des cartes de transputers (T800), qui divisent nos temps de calcul d'une manière appréciable [5], il faut bien réfléchir avant de calculer sur une grosse machine. Pour nos applications, 1 T800 est à peu près deux fois plus rapide qu'un PC équipé d'un processeur Intel 80386 $(20 \mathrm{MHz})$ et d'un accélérateur Weitek mW1167. 2 T800 sont aussi rapides qu'un Sun 3 et 4 T800 sont équivalents à un SUN 4 et aussi au Cyber 855 . Les programmes ne seront probablement pas installés sur Cray X/MP, car ce "super ordinateur» est équivalent à environ 50 transputers [7]. Lorsque l'on étudie les coûts $(1 \mathrm{~T} 800 / 20 \mathrm{MHz}$ coûte moins de 300 US \$), des cartes (type AT, une fente de PC de hauteur) fabriquées à Zurich [13] avec 9 T800 $(25 \mathrm{MHz})$ et $28 \mathrm{MB}$ de mémoires $(70 \mathrm{~ns})$ sont à moins de 10000 US \$), la tentation de s'équiper d'une machine à "utilisateur unique " devient grande. Notre groupe y a succombé et s'évite ainsi des transferts de données coûteux en temps et en fatigue nerveuse. Et puis plus il y a de mémoire à disposition plus grand peut être le nombre d'inconnues, par exemple $16 \mathrm{MB}$ autorisent plus de 1400 inconnues. Les cartes de transputers ont une structure de pipeline qui est parfaite pour notre application, car pour l'inversion de la matrice triangulaire, seul un aller et retour est nécessaire. Les communications entre transputers sont donc réduites à un minimum.

Le fait de n'avoir à discrétiser que le pourtour apporte une simplification notable pour l'entrée des données. C'est un des avantages de la méthode qui s'adapte particulièrement bien aux PC. D'autre part, l'utilisation des multipôles dans (ou plus exactement à l'extérieur de) chaque domaine permet de « zoomer » dans ces domaines avec des routines très simples. Ces routines aussi bien d'entrée que de sortie de données sont très importantes. En effet, un éditeur graphique qui permet de construire des géométries complexes et de les corriger en cas d'erreur, autorise un confort important pour l'utilisateur. Il ne faut pas se leurrer et un éditeur graphique demande toujours un peu d'habitude pour s'en servir rapidement, mais c'est quand même plus fiable que de taper une série de chiffres exprimant la géométrie. Actuellement, il existe un éditeur graphique en $2 \mathrm{D}$ et en $3 \mathrm{D}$ sur Sun 3 et pour les PC. L'utilisation de la couleur permet une meilleure visualisation des volumes.

Quant aux programmes de sortie, ils sont encore bien plus importants car ils permettent de jauger la qualité des résultats. Comme la méthode permet de se rapprocher très près de la surface des corps, il est facile de contrôler si les champs possèdent la configuration qu'ils devraient avoir (par exemple, à la surface d'un métal parfait, le champ électrique devrait uniquement posséder une composante normale, etc.). C'est, en fait, le moyen le plus efficace de contrôle que nous ayons (bien sûr, une erreur sur le pourtour trop grande est certainement un signe que le calcul n'est pas bon, mais il peut y avoir des cas où l'erreur est petite et où le champ n'est pourtant pas réaliste). Les exemples que nous présenterons ont été calculés à l'aide des programmes suivants, dont les caractéristiques communes sont une dépendance périodique du temps ainsi que la linéarité, l'homogénéité, l'isotropie de tous les (sous-)domaines.

Le programme à deux dimensions (symétrie cylindrique) possède des routines de représentation des données de sortie qui permettent de représenter la valeur du champ sous forme de flèches ou de lignes iso (en $2 \mathrm{D}$ et $3 \mathrm{D}$ ) ou encore les surfaces d'intensité. On peut bien évidemment séparer chaque composante pour la représenter. Il résout les problèmes de diffraction et de conduction (valeurs propres).

Le programme à trois dimensions [1] résout les problèmes de diffraction avec ou sans symétrie 

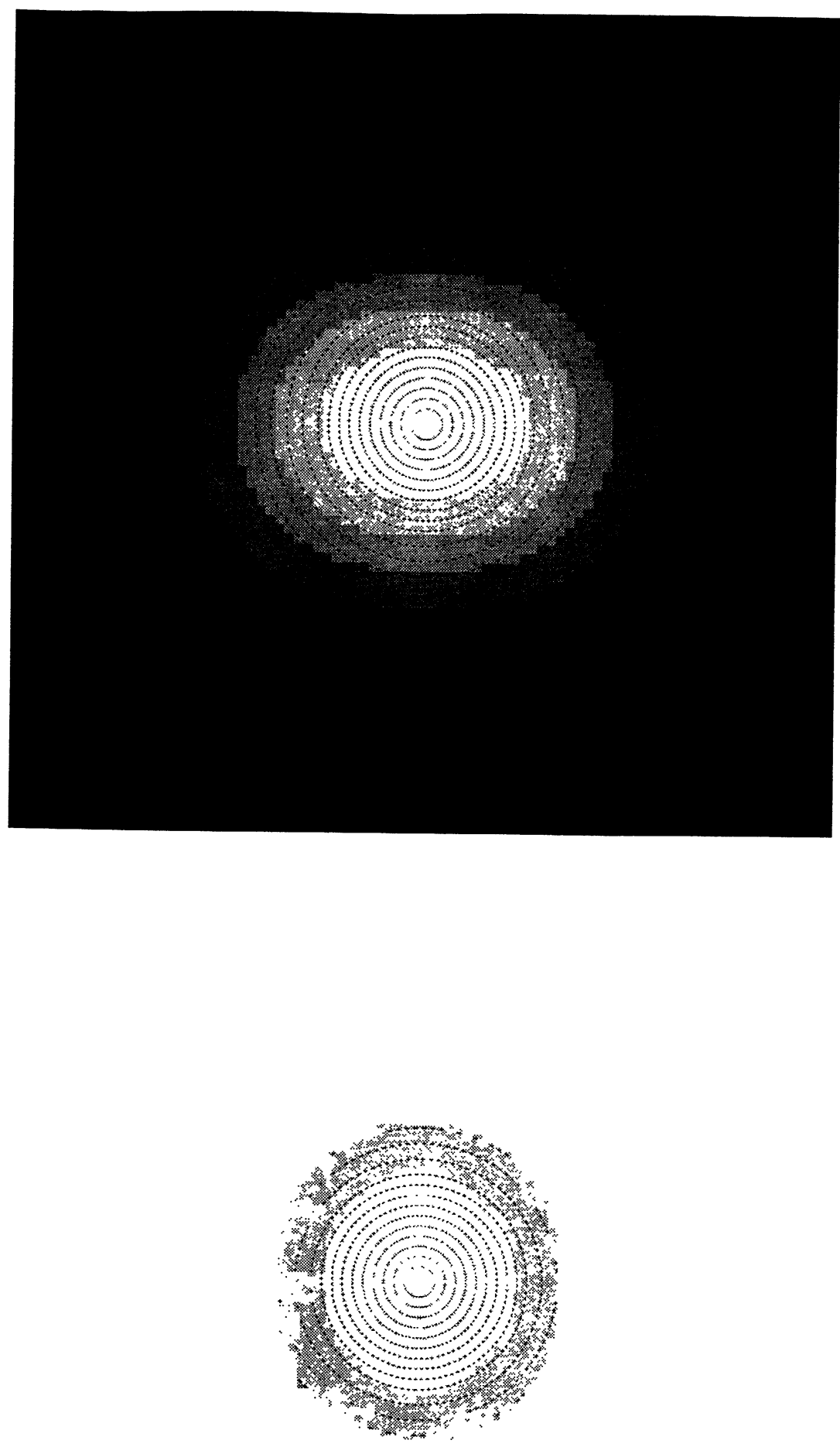

Fig. 3. - a) Champ magnétique d'une fibre optique circulaire divisée en 21 domaines. b) Champ Poynting d'une fibre optique circulaire divisée en 21 domaines.

[a) Magnetical field of a circular optical fiber subdivised in 21 domains. b) Poynting field of a circular optical fiber subdivised in 21 domains.] 
rotationnelle. Il permet aussi de calculer les jonctions 2D-3D, car toutes les fonctions du code $2 \mathrm{D}$ sont implantées.

2. Applications. - Trois exemples vont être présentés, deux à $2 \mathrm{D}$ et un en $3 \mathrm{D}$ :

- un guide d'onde lumineux dont l'indice de réfraction du matériau est une fonction à pas discret du rayon; fente ;

- un train d'impulsions passant à travers une

- diffraction d'une onde plane sur un esquimau dans son igloo.

Ces exemples démontrent les possibilités actuelles des programmes. Pour les exemples à 2D, les dimensions de la géométrie ainsi que les fréquences sont normées. Ce qui importe ici, c'est la relation entre la longueur d'onde et la dimension du corps.
2.1 Guide d'onde. - Une fibre optique circulaire dont l'indice de réfraction varie de 1 à $2 \quad(1 \leqslant$ $\left.\varepsilon_{\mathrm{R}} \leqslant 4\right)$ est divisée en 20 domaines à la frontière desquels la permittivité relative saute de 0,1 à 0,3 suivant les domaines. Le diamètre de la fibre est de 4 fois la longueur d'onde. La forme convient très bien au programme et seuls 2 à 3 points sont nécessaires sur le pourtour des domaines. Ce problème a été calculé sur Toshiba 5200 en moins de $10 \mathrm{~min}$ et la résolution totale, y compris l'entrée et la sortie des données (sous forme des Figs. 3) ne prend pas plus de $15 \mathrm{~min}$. La plus grande partie du temps est consacrée à calculer la constante de propagation $\gamma \simeq 1,327$, l'inversion de la matrice avec aussi peu de coefficients ne prenant qu'un peu plus d'une minute ! Les figures 3 décrivent l'intensité du champ magnétique et le champ Poynting (clair pour les valeurs les plus élevées). Les pointillés délimitent chaque domaine.

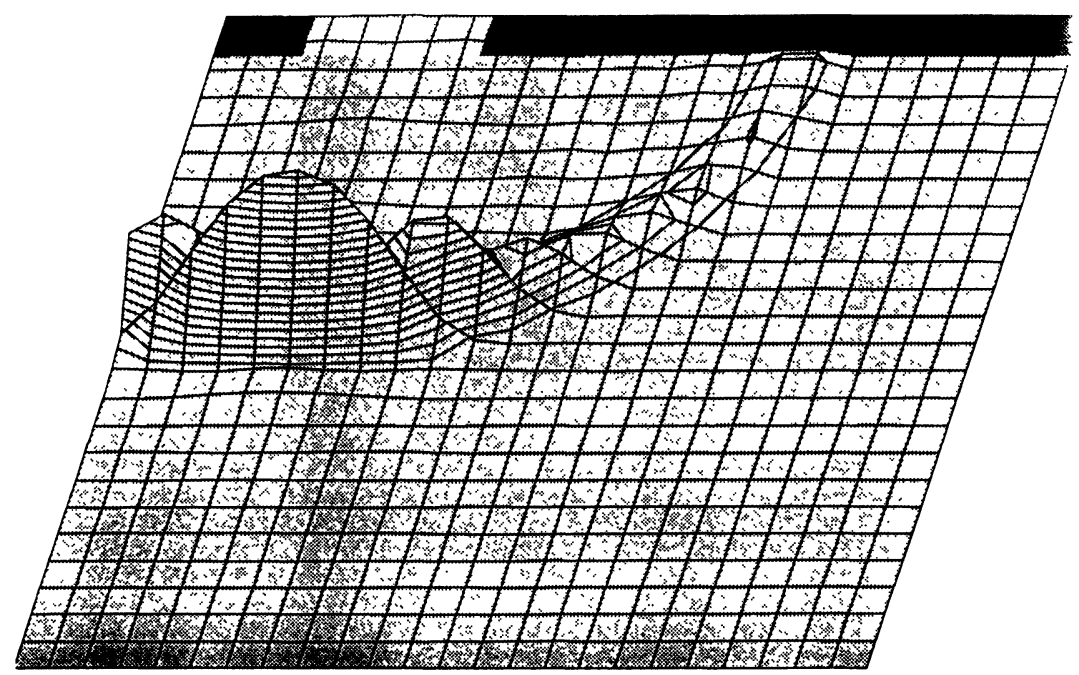

Fig. 4. - Intensité de l'énergie électromagnétique pour une impulsion à travers une fente.

[Intensity of the electromagnetical energy for a pulse through an opening.]

2.2 Train d'impulsions. - Ce second exemple teste le développement du programme pour la transformation de Fourier. Tout d'abord, un train d'onde dans l'espace vide a été calculé pour vérifier la valeur de la transformation. Dans le cas des séries de Fourier, il est possible de choisir le nombre de fréquences que l'on désire calculer ainsi que la largeur de l'impulsion (qui détermine la plus haute fréquence) et de la distance entre deux impulsions. Ces choix s'effectuent en fonction de la géométrie et des souhaits de l'utilisateur en tenant compte de la possibilité d'effacements de digits si les impulsions sont trop éloi- gnées. Dans notre cas, 25 fréquences ont été calculées, dont la plus haute était de $52 \mathrm{MHz}$ avec un espacement de 18 pas de temps sur 20 que compte une période. Puis, ces impulsions ont dû passer à travers une fente (2D) moins large que leurs amplitudes. C'est l'énergie électromagnétique qui est représentée à la figure 5. La partie sombre représente le pourtour métallique.

Pour étalonner le programme encore plus exactement, une loupe a été placée devant l'ouverture et finalement un cylindre semi-conducteur au foyer de la loupe. Les figures 5 donnent un aperçu du 

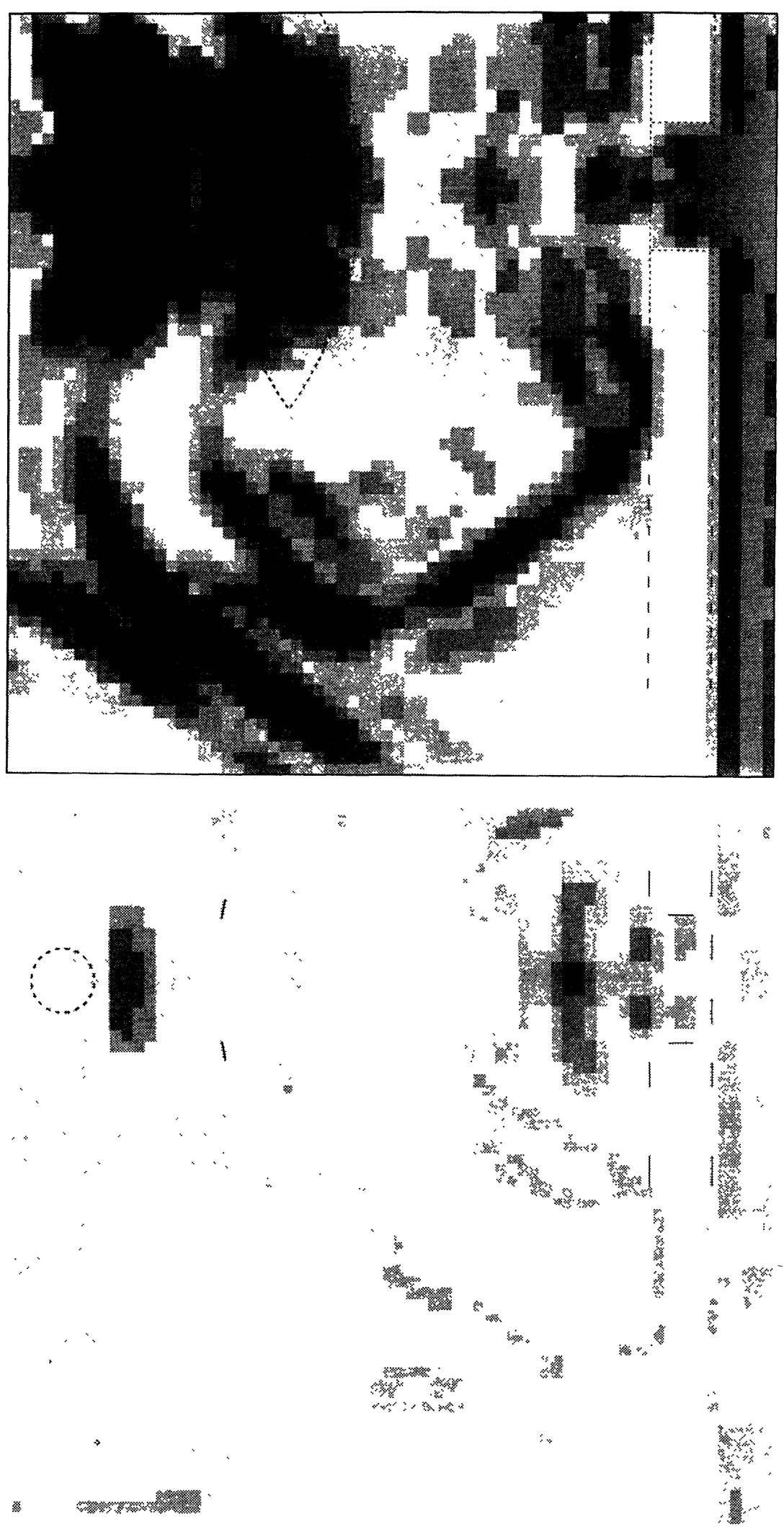

Fig. 5. - a) Intensité de l'énergie électromagnétique d'un train d'onde sur une loupe avec cylindre placé au foyer pour des instants différents. b) Intensité de l'énergie électromagnétique d'un train d'onde sur une loupe avec cylindre placé au foyer pour des instants différents.

(a) Intensity of the electromagnetical energy for a series of pulse on a lens with a lossy cylinder placed at the focus for different times. b) Intensity of the electromagnetical energy for a series of pulse on a lens with a lossy cylinder placed at the focus for different times.] 


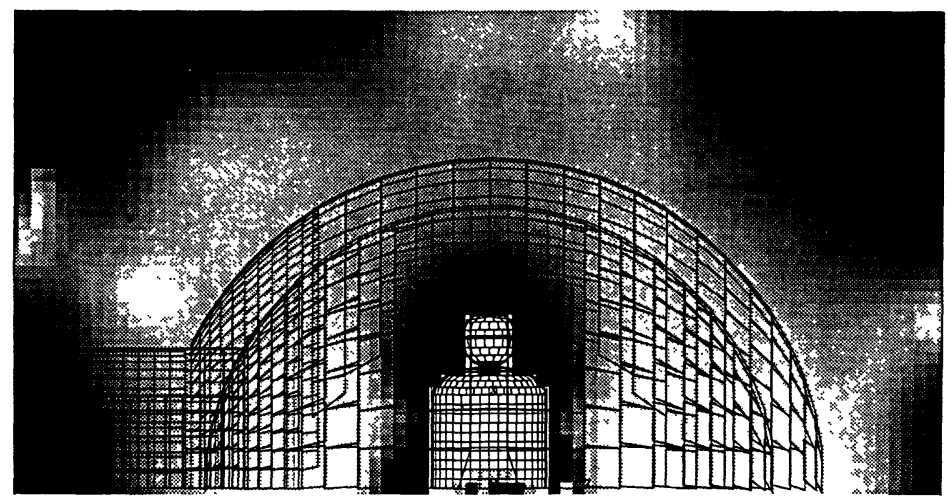

Fig. 6. - Champ Poynting moyen pour un esquimau dans un igloo éclairé par une onde plane (noir pour les plus fortes amplitudes).

[Time averaged Poynting field for an eskimo in an igloo illuminated by a plane wave (black for bigger amplitude).]

comportement de l'énergie électromagnétique pour des instants différents.

On remarquera dans la figure 6 les nombreuses réflexions.

2.3 Esquimau dans son igloo. - Cet exemple n'a pas d'applications directes, sauf si l'on considère le problème plus général de l'influence des champs électromagnétiques sur les corps humains dans un environnement complexe. Par sa géométrie, c'est un problème à $3 \mathrm{D}$ avec 2 plans de symétries (le sol est considéré parfait conducteur), une ouverture et des discontinuités. Les matériaux choisis (glace et tissus humains) ont les caractéristiques suivantes :

- glace :

$$
\varepsilon_{\mathrm{R}}=1 \quad \mu_{\mathrm{R}}=1 \quad \sigma=1,0 \mathrm{~m}[\Omega \mathrm{m}]^{-1} .
$$

- tête du mannequin :

$$
\varepsilon_{\mathrm{R}}=55 \quad \mu_{\mathrm{R}}=1 \quad \sigma=1,4 \mathrm{~m}[\Omega \mathrm{m}]^{-1} .
$$

- corps du mannequin :

$$
\varepsilon_{\mathrm{R}}=45 \quad \mu_{\mathrm{R}}=1 \quad \sigma=0,9 \mathrm{~m}[\Omega \mathrm{m}]^{-1} .
$$

La fréquence est de $50 \mathrm{MHz}$ pour une hauteur d'igloo de 3,50 m. L'esquimau est grand $(1,85 \mathrm{~m})$. L'onde plane arrive horizontalement du côté de l'entrée avec une polarisation horizontale (le champ électrique est parallèle au sol). Ce problème repré- sente plus de 700 inconnues pour 7000 équations, mais il peut être résolu en plusieurs pas séparés. Tout d'abord l'igloo seul a été calculé, puis le résultat a été utilisé comme source du problème avec esquimau. Bien entendu, le mannequin a été aussi calculé séparément pour contrôler la validité du modèle. C'est la même manière d'agir que pour la fente avec la loupe et le cylindre au point de focalisation.

\section{Conclusion.}

Une méthode de résolution pour le calcul des champs électromagnétiques aussi bien en $2 \mathrm{D}$ qu'en $3 \mathrm{D}$ avec une dépendance périodique du temps a été présentée. Son efficacité a été confirmée par le développement de codes de calcul efficaces qui ont pu être facilement installés sur des PC. Afin d'augmenter encore les performances des codes, le travail actuel est dédié à l'implantation de différentes techniques déjà existantes dans d'autres méthodes. Ceci pour permettre d'élargir l'éventail des champs d'applications aux calculs de matériaux non linéaires 'ou à des objets très fins.

\section{Remerciements.}

Tout particulièrement à $\mathrm{Ch}$. Hafner pour ses conseils éclairés, et les figures 4 et 5 ainsi qu'à L. Bomholt pour le soutien de ses programmes. 


\section{Bibliographie}

[1] Bomholt L. and Hafner Ch., A MMP Program for Computations of 3D Electromagnetic Fields on PC's, Proc. of the 5th Ann. Rev. of ACES (Monterey, CA) March 21-23, 1989.

[2] HAFNer Ch., Beiträge zur Berechnung der Ausbreitung Elektromagnetischer Wellen in Zylindrischen Strukturen mit Hilfe des "Point Matching ", Verfahrens, Thèse ETH Nr. 6683, Zürich, 1980.

[3] HAFNER Ch., Numerische Berechnung Elektromagnetischer Felder (Springer Verlag, Berlin) 1987.

[4] HAFNER Ch., Computations of electromagnetic fields by the MMP method, Proc. of URSI on EM theory, Stockholm (Aug. 14-17, 1989).

[5] Hafner Ch. and Kiener St., Parallel Computations of 3D Electromagnetic Fields on Transputers, ISAP, Tokyo (Aug. 22-25, 1989).

[6] HARRINGTON R., Field Computation by Moment Methods (New York: MacMillan) 1968.

[7] HofmaN J., Transputers Applications in Numerical physics, ISPRA courses: Parallel Processing with PC, CECJR (June 5-9, 1989).

[8] KIENER S., La méthode MMP appliquée à la CEM, $5^{\mathrm{e}}$ colloque int. en langue française sur la CEM, Evian, 12-14 sept. 1989, CIII-2.

[9] KIENER S., Bodies with sharp Edges : Calculation of Near- and Farfields, Proc. IEEE AP's Int. Symp. and URSI R.S.M., San Jose USA (June 1989).

[10] KIENER S., Eddy currents in Bodies with Sharp Edges by the MMP-method, Compumag, Tokyo (Sept. 3-7, 1989).

[11] Kiener S. and Li J., On the solution of Periodical Structures with the GMT, Proc. IEEE AP's Int.
Symp. and URSI R.S.M., Dallas USA (May 1990).

[12] KLAUS G., 3D Streufeldberechnungen mit Hilfe der MMP Methode, Thèse ETH Nr. 7792, Zürich (1985).

[13] Lehareinger Y., Parallel Processing in the Field of Biomedical Engineering, ISPRA courses : Parallel Processing with PC, CECJR (June 5-9, 1989).

[14] Ludwig A., A new technique for numerical electromagnetic, IEEE AP's Newsletter 31 (1989) 4041.

[15] Leuchtmann P., A Completely Automated Procedure for the Choice of Functions in the MMP Method, Computational Methods in flow Analysis, Vol. 1 (Okayama University of Science) 1988.

[16] SARKAR T. K., From "Reaction concept" to «Conjugate Gradient»: Have we made any progress? IEEE AP's Newsletter 31 (1989) 6-12.

[17] Singer H. et al., A Charge Simulation Method for the Calculation of High-Voltage Fields, IEEE Trans. on Power Appar. Syst. 93 (1974) 16601668.

[18] Vekua I. N., New methods for solving Elliptic Equations (North-Holland Publ. Comp., Amsterdam) 1967.

[19] Zhang W. X., The Methodology of Boundary Methods, Proc. IEEE AP's Int. Symp. and URSI R.S.M., Syracuse USA (June 1988) 299302.

[20] ZhANG W. X., Methodology in Electromagnetic Boundary Value Problems, Kolloquium über " Elektrotechnik und Nachrichtentechnik », ETH-Zürich (Jan.-Feb. 1989). 